\title{
Study of the correlation between H-ras mutation and primary hepatocellular carcinoma
}

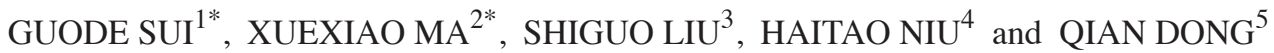 \\ Departments of ${ }^{1}$ Emergency General Surgery and ${ }^{2}$ Spinal Surgery; ${ }^{3}$ Gout Laboratory; \\ Departments of ${ }^{4}$ Urology and ${ }^{5}$ Pediatric Surgery, The Affiliated Hospital of Medical College, Qingdao University, \\ Qingdao 266003, P.R. China
}

Received April 9, 2012; Accepted July 20, 2012

DOI: $10.3892 / \mathrm{ol} .2012 .832$

\begin{abstract}
The aim of this study was to investigate the correlation between H-ras mutation and primary hepatocellular carcinoma (HCC) and to describe the role of $\mathrm{H}$-ras mutation in carcinogenesis. Clinical samples of 69 patients were collected and the expression levels of H-ras in HCC and the surrounding normal tissues were examined using HotStarTaq PCR. $\mathrm{H}$-ras mutation was further analyzed using the PCR direct sequencing method. The results showed that H-ras mutation was present in 49 samples $(49 / 69,71.01 \%)$, of which 19 had codon 40 mutated from CTA to CTG and 30 had codon 61 mutated from GGC to AGC. By contrast, only 2 mutations were found in the normal tumor-adjacent tissues. The H-ras mutation rate in the high-risk of metastatic recurrence group was markedly higher than that in the low-risk group $(\mathrm{P}<0.01)$. The H-ras mutation rate in patients with metastatic recurrence during postoperative follow-up was also significantly higher than that in patients without metastatic recurrence $(\mathrm{P}<0.01)$. Based on the above results, the H-ras mutation frequency in cancer tissues is markedly higher compared with that in normal tissues. H-ras mutation may play an important role in the genesis and development of HCC and may serve as a reliable marker for individual comprehensive therapy in HCC.
\end{abstract}

Correspondence to: Professor Qian Dong, Department of Pediatric Surgery, The Affiliated Hospital of Medical College, Qingdao University, 16 Jiangsu Road, Qingdao 266003, P.R. China

E-mail: qyqian_dong@126.com

Professor Haitao Niu, Department of Urology, The Affiliated Hospital of Medical College, Qingdao University, 16 Jiangsu Road, Qingdao 266003, P.R. China

E-mail: niuhaitao1976@126.com

*Contributed equally

Key words: primary hepatocellular carcinoma, HotStarTaq PCR, $\mathrm{H}$-ras, mutation, sequencing

\section{Introduction}

Primary hepatocellular carcinoma (HCC) is a common malignant tumor, particularly in China and Southeast Asia (1). Although the resection rate of HCC has improved in the last 20 years, the general therapeutic efficacy is not yet satisfactory. The mortality rate of HCC ranks second in all malignant tumors in China, due to postoperative metastatic recurrence (2). Currently, the molecular basis for the characteristics of HCC is unknown and there is a need to identify the cancer biology and develop new therapeutic strategies.

Ras proteins have essential roles in controlling the activity of multiple downstream effector pathways that regulate normal cellular proliferation (3). Ras-controlled pathways modulate several transcription factors that eventually link Ras activity to various phases of the cell cycle. As an impotant member of the Ras family, H-ras mutation has been widely investigated in multiple types of solid tumor. Over the past decade, H-ras has emerged as a significant target for anticancer therapies and has become a hotspot of anticancer research, while the exact mechanism remains unknown (4).

In the present study, HotStarTaq PCR was adopted to examine the expression of H-ras in HCC tissues and normal tissues adjacent to the tumor, and $\mathrm{H}$-ras mutation was analyzed using PCR direct sequencing. Based on our findings, the essential role of H-ras in the diagnosis and treatment of HCC patients is discussed, especially in the Chinese population.

\section{Materials and methods}

Samples. A total of 69 paired HCC and normal liver samples (confirmed by two individual pathological diagnoses) were obtained from patients treated at the The Affiliated Hospital of Medical College, Qingdao University (Qingdao, China) for HCC. No patient had distant metastatic disease at surgery and no patient had been treated previously with intravesical chemotherapy. For the selection of surrounding non-tumor liver tissues, specimens were obtained from tissues at a clear distance from the edge of tumors $(>1 \mathrm{~cm})$, if there was no evidence of nearby tumor invasion. The cancer tissue and the adjacent microscopically normal samples were rinsed in sterile PBS and snap frozen in liquid nitrogen immediately after removal. The research protocol was approved by 
the Institutional Review Board and informed consent was obtained from patients. The average age of the patients in this study, including 42 males and 27 females, was between 22 and 73 years $(57.9 \pm 13.4)$. Of the 69 patients, the greatest diameter of the tumor was $>5 \mathrm{~cm}$ in 48 cases and $5 \mathrm{~cm}$ in 21. Tumor capsules were integrated in 32 and disintegrated in 37 cases. Serum AFP was positive in 40 and negative in 29; HBsAg was positive in 38 and negative in 31. Liver cirrhosis was detected in 34 cases. A total of 24 patients had Edmondson grade I or II disease and the remaining 45 patients had Edmondson grade III or IV. A total of 28 patients had TNM stage I or II disease and 41 had TNM stage III or IV. According to the operative records and postoperative pathological data, $36 \mathrm{HCC}$ patients with cancer emboli, intrahepatic dissemination (satellite foci or multiple nodules) and/or lymph node metastasis were classified as high-risk of metastatic recurrence and the remaining 33 patients without emboli, dissemination and/or metastasis were described as low-risk of metastatic recurrence. A total of 63 patients were followed up for 16-35 months after surgery, during which time neoplastic metastasis or recurrence was found in 38 patients.

RNA extraction and cDNA synthesis. Approximately $100 \mathrm{mg}$ tumor or liver tissue was used for total RNA isolation using TRIzol reagent (Gibco-BRL, Carlsbad, CA, USA) according to the manufacturer's instructions. First-strand cDNA was synthesized using $5 \mu \mathrm{l}$ total RNA with oligo (dT) 16 primer in a $50-\mu 1$ reverse transcription mixture containing $10 \mu \mathrm{l}$ of $5 \mathrm{X}$ first strand buffer, $2.5 \mu \mathrm{l}$ dNTP mixture containing $25 \mathrm{mmol} / \mathrm{l}$ of each deoxynucleotide triphosphate base (Pharmacia Biotech Co., Tokyo, Japan), $2.5 \mu$ l ribonuclease inhibitor (Takara Biochemicals, Ohotsu, Japan), $25 \mu 1$ dd $_{2} \mathrm{O}$ (managed with DEPC in advance) and $2.5 \mu \mathrm{l}$ avian myeloblastosis virus reverse transciptase (Takara Biochemicals).

Primer design. The resulting cDNA was used for PCR amplification using Taq polymerse (Takara Biochemicals). The sequence of the H-ras gene open reading frame was obtained from the gene bank and the primer was designed using Primer Express 2.0 (Applied Biosystems, Foster City, CA, USA). The upstream primer is before ATG, H1=5' - GGCAGGAGACCCTGTAGGAG-3'; the downstream primer was located at exon 2, H2=5'-GGTTCACCTGTACTGGTGGAT-3'; the amplification area included exons 1 and 2, totaling $613 \mathrm{bp}$. The primer was synthesized by Shanghai Sangon Biological Engineering Technology \& Service Co., Ltd. (Shanghai, China) The PCR conditions included initial denaturation at $95^{\circ}$ for $15 \mathrm{~min}$, followed by 40 cycles of amplification with subsequent denaturation at $94^{\circ} \mathrm{C}$ for $60 \mathrm{sec}$, annealing at $60^{\circ} \mathrm{C}$ for $60 \mathrm{sec}$ and extension for $60 \mathrm{sec}$ at $72^{\circ} \mathrm{C}$. The amplified products were examined by agarose gel electrophoresis, and recorded by a motored molecular imaging system. A total volume of 101 of PCR products underwent electrophoresis using $12 \mathrm{~g} / \mathrm{l}$ agarose gel and were visualized by UV absorption and ethidium bromide.

Sequencing. DNA sequencing was performed on an ALF express DNA automatic sequencer (Pharmacia Biotech Co.) by

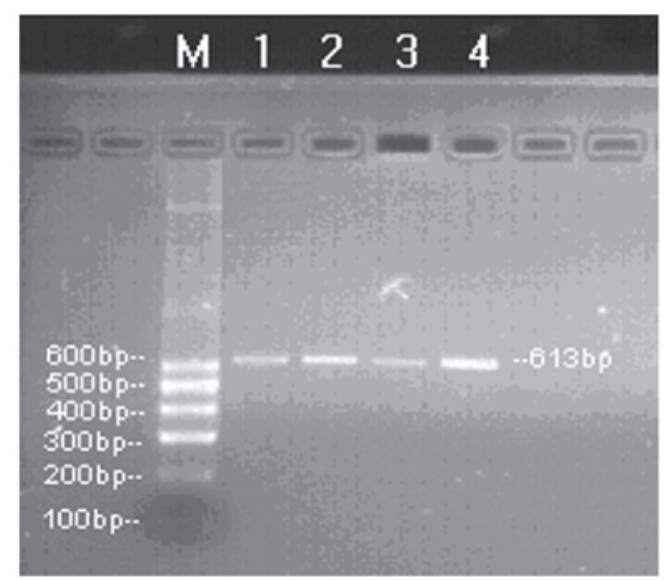

Figure 1. Expression of H-ras mRNA in clinical samples of HCC. From the top, M [DNA Marker (DL2000)] indicates 600, 500, 400, 300, 200 and $100 \mathrm{bp}$, successively. The clear electrophoretic band at $600 \mathrm{bp}$ is the PCR product of the sample. HCC, hepatocellular carcinoma.

the dideoxy terminal termination method. The sequenced HPA segment was $613 \mathrm{bp}$ in length. The sequence of the amplified $\mathrm{H}$-ras segment was compared with the gene bank database and analyzed for homogeneity using the NCBI BLAST program.

Statistical analysis. The significance of the differences between two groups was tested using Chi-square analysis. $\mathrm{P}<0.05$ was considered to indicate a statistically significant result.

\section{Results}

Expression of H-ras mRNA in HCC. H-ras mRNA was amplified in all the clinical samples. Electrophoretic analysis showed a bright band $\sim 600$ bp in length in these patients. The electrophoresis band was clear and there was no dispersive band, indicating its high specificity (Fig. 1).

Sequencing. After the sequence of the PCR products of the 69 samples of HCC was examined, the H-ras gene was found to be mutated in 49 samples of HCC tumor tissue; 19 samples had a mutation in codon 40 from CTA to CTG; 30 had a mutation in codon 61 of GGC to AGC. By contrast, only 2 mutations were found in the samples of normal tissues adjacent to the cancer. The sequencing maps are shown in Fig. 2. The H-ras mutation rate in the tumor tissues of HCC was $71.01 \%(49 / 69)$ and was significantly higher than that in the surrounding nontumor liver tissues $(\mathrm{P}<0.01)$.

Correlation between H-ras mutation and clinicopathological indices. By statistical analysis, no significant correlation of the $\mathrm{H}$-ras mutation rate was identified with tumor size, capsule, AFP, HBsAg or liver cirrhosis (all P>0.05; Table I). The $\mathrm{H}$-ras mutation rate in the Edmondson grade I or II group was significantly lower than that in Edmondson grade III or IV group $(\mathrm{P}<0.01)$ and the rate in TNM stage I or II group was also markedly lower than that in TNM stage III or IV group $(\mathrm{P}<0.01$; Table I). 
Table I. Correlation between H-ras mutation and the clinicopathological parameters of HCC.

\begin{tabular}{|c|c|c|c|c|}
\hline Parameter & $\mathrm{n}$ & $\begin{array}{c}\text { H-ras } \\
\text { mutation }\end{array}$ & $\begin{array}{l}\text { H-ras } \\
\text { normal }\end{array}$ & P-value \\
\hline \multicolumn{5}{|c|}{ Size of tumor $(\mathrm{cm})$} \\
\hline$>5$ & 48 & 37 & 11 & 0.09 \\
\hline$\leq 5$ & 21 & 12 & 9 & \\
\hline \multicolumn{5}{|l|}{ Tumor capusle } \\
\hline Integrated & 32 & 26 & 6 & 0.08 \\
\hline Disintegrated & 37 & 23 & 14 & \\
\hline \multicolumn{5}{|l|}{ AFP } \\
\hline Positive & 40 & 28 & 12 & 0.82 \\
\hline Negative & 29 & 21 & 8 & \\
\hline \multicolumn{5}{|l|}{ HBsAG } \\
\hline Positive & 38 & 23 & 15 & 0.07 \\
\hline Negative & 31 & 26 & 5 & \\
\hline \multicolumn{5}{|l|}{ Liver cirrhosis } \\
\hline Yes & 34 & 24 & 10 & 0.94 \\
\hline No & 35 & 25 & 10 & \\
\hline \multicolumn{5}{|c|}{ Edmondson grade } \\
\hline I, II & 24 & 9 & 15 & $<0.01$ \\
\hline III, IV & 45 & 40 & 5 & \\
\hline \multicolumn{5}{|l|}{ TNM staging } \\
\hline $\mathrm{I}, \mathrm{II}$ & 28 & 11 & 17 & $<0.01$ \\
\hline III, IV & 41 & 38 & 3 & \\
\hline
\end{tabular}

HCC, hepatocellular carcinoma.

Correlation between $H$-ras mutation and metastatic recurrence of $\mathrm{HCC}$. The H-ras mutation rate in the high-risk of metastatic recurrence group was markedly higher than that in the low-risk group $(\mathrm{P}<0.01)$ and the rate in the metastatic recurrence group was also significantly higher than that in the non-metastatic recurrence group $(\mathrm{P}<0.01$; Table II).
Table II. Correlation between H-ras mutation and metastatic recurrence of $\mathrm{HCC}$.

\begin{tabular}{lcccc}
\hline Group & $\mathrm{n}$ & $\begin{array}{c}\text { H-ras } \\
\text { mutation }\end{array}$ & $\begin{array}{c}\text { H-ras } \\
\text { normal }\end{array}$ & P-value \\
\hline $\begin{array}{l}\text { Risk of metastatic } \\
\text { recurrence }\end{array}$ & 36 & 32 & 4 & $<0.01$ \\
$\quad$ High & 33 & 17 & 16 & \\
Low & & & & \\
Metastatic & & & & \\
recurrence & 38 & 35 & 3 & $<0.01$ \\
$\quad$ Yes & 31 & 14 & 17 & \\
No & & & & \\
\hline HCC, hepatocellular carcinoma. & & & \\
\hline
\end{tabular}

\section{Discussion}

The H-ras gene is located at 11p15.5. The possible carcinogenic mechanism of H-ras is as follows: the mutated H-ras gene results in overexpression of the H-ras protein. The $\mathrm{H}$-ras protein inhibits the activation of a nuclease which triggers apoptosis and leads directly to a significant decrease in cell death (5). It has been found that ras mutation occurs in numerous types of human tumors and that the mutation rate of the ras genes can reach $90 \%$ in pancreatic, $40-50 \%$ in colon, $40 \%$ in lung and $37 \%$ in bladder cancer (5). The present study showed that H-ras mutation occurred in 49 samples $(49 / 69$, $71.01 \%$ ), of which 19 had a mutation in codon 40, of CTA to CTG, and 30 had a mutation in codon 61, of GGC to AGC. Compared with the cancer samples, H-ras mutation was found in only 2 normal tissues adjacent to the cancer. Our results indicate that $\mathrm{H}$-ras mutation may be a reliable biomarker in HCC.

No correlation was found between $\mathrm{H}$-ras mutation and the integrity of the tumor capsule or tumor size in this study. In addition, $\mathrm{H}$-ras mutation was not found to be associated

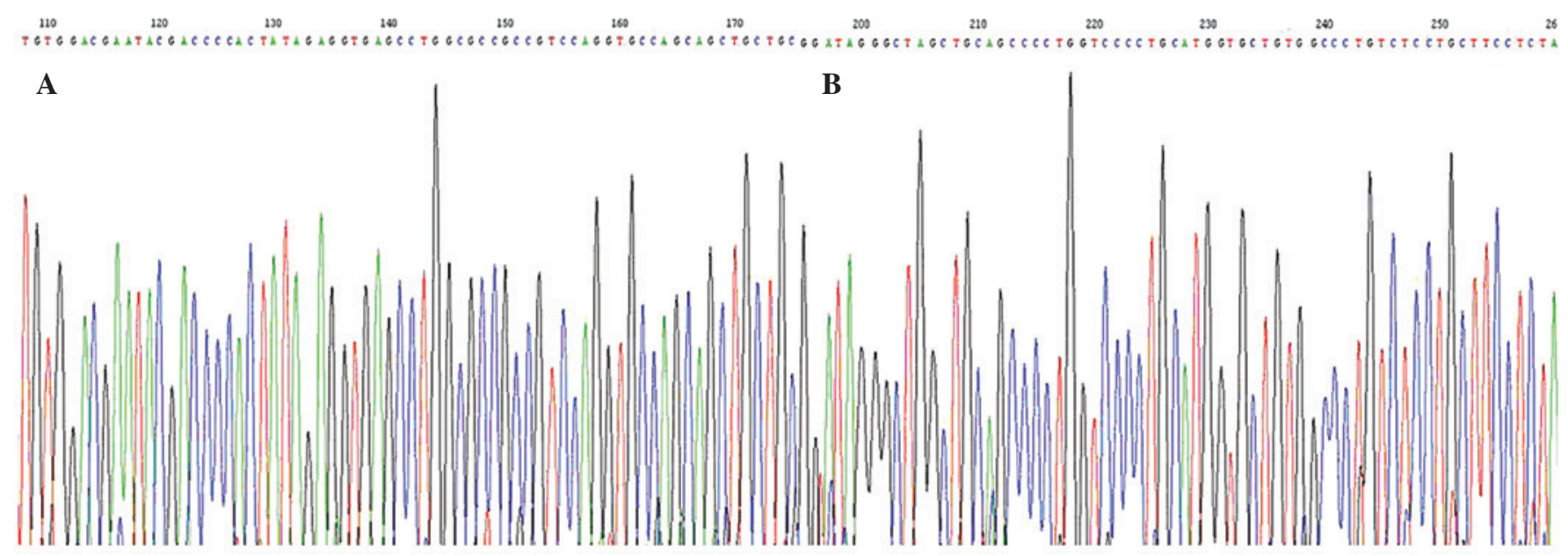

Figure 2. Sequencing maps. (A) Codon 40 mutated from CTA to CTG; (B) codon 61 mutated from GGC to AGC. 
with AFP, HBsAg or liver cirrhosis. H-ras mutation was not correlated with $\mathrm{HBsAg}$, indicating that $\mathrm{H}$-ras mutation may be used for the diagnosis of HCC patients infected with HBV, as is the case of most HCCs in China. It has been reported that $\mathrm{H}$-ras mutation in cancerous tissues is closely correlated with tumor invasion, metastasis and angiogenesis $(6,7)$. Both metastatic recurrence and tumor microvessel density (MVD) in tumors may significantly increase with H-ras mutation (8). In this study, the mutation rate in HCCs was $71.01 \%$ and H-ras mutation was associated with the pathological classification and TNM stage, which were similar to the conclusions drawn by a previous study (9). The marked difference in H-ras mutation between the low- and high-risk of metastatic recurrence groups in our study indicated that $\mathrm{H}$-ras mutation was associated with the invasion and metastasis of HCC. The significant difference between the metastatic and non-metastatic recurrence groups in our study was also similar to the results of a previous study (6). This result further showed that $\mathrm{H}$-ras mutation was associated with metastatic recurrence and that there was a higher degree of invasiveness and risk of postoperative recurrence in patients with $\mathrm{H}$-ras mutation compared with normal $\mathrm{H}$-ras patients, and that $\mathrm{H}$-ras mutation may provide a potential and valuable index to predict postoperative metastatic recurrence (10).

Previous studies have reported that $\mathrm{H}$-ras mutation may be an effective marker in cancer diagnosis and molecular therapy (11). The exact mechanism remains unknown. Certain studies have reported the levels of growth factors, including transforming growth factor- $\beta$ (TGF- $\beta$ ) and fibroblast growth factor (bFGF), enhanced DNA synthesis and anchorage-dependent growth in cells with H-ras mutation $(12,13)$. Furthermore, $\mathrm{H}$-ras mutation caused progressive tumors in nude mice; however, those tumors were distinct from v-ras-induced tumors, indicating that H-ras mutation was directly involved in cell growth and transformation and the development of tumors (14). Although the present study offers limited information with regard to the possible correlation between H-ras mutation and cancer, we may offer extra information concerning the diagnosis and treatment of $\mathrm{HCC}$, especially in China. Being a developing country, most Chinese patients lack medical insurance and the cost of adjuvant therapy is a problem. The ongoing debates that have not been resolved in China are how to identify patients who have the highest risk of suffering recurrence and how to increase the cost efficiency of disease management (15). According to the above results, $\mathrm{H}$-ras mutation was not a early event in carcinogenesis and is associated with invasion and metastatic recurrence, which may be especially significant in the selection of apporiate candidates for perioperative comprehensive therapy.

In conclusion, the detection of H-ras mutation has the potential to aid an improved understanding of $\mathrm{HCC}$ and to offer extra information concerning the diagnosis and treatment of HCC.

\section{Acknowledgements}

This study was supported by a grant from the Natural Science Foundation of Shandong Province, China (Y2005C45).

\section{References}

1. Luk JM and Liu AM: Proteomics of hepatocellular carcinoma in Chinese patients. OMICS 15: 261-266, 2011.

2. Yang T, Zhang J, Lu JH, Yang LQ, Yang GS, Wu MC and Yu WF: A new staging system for resectable hepatocellular carcinoma: comparison with six existing staging systems in a large Chinese cohort. J Cancer Res Clin Oncol 137: 739-750, 2011.

3. Davies M, Prime SS, Eveson JW, Price N, Ganapathy A, D'Mello A and Paterson IC: Transforming growth factor- $\beta$ enhances invasion and metastasis in Ras-transfected human malignant epidermal keratinocytes. Int J Exp Pathol 93: 148-156, 2012 .

4. Gus-Brautbar Y, Johnson D, Zhang L, Sun H, Wang P, Zhang S, Zhang L and Chen YH: The anti-inflammatory TIPE2 is an inhibitor of the oncogenic Ras. Mol Cell 45: 610-618, 2012.

5. Overmeyer JH and Maltese WA: Death pathways triggered by activated Ras in cancer cells. Front Biosci 16: 1693-1713, 2011.

6. Cullen JM, Williams C, Zadrozny L, Otstot JT, Solomon GG, Sills RC and Hong HH: H-ras consensus sequence and mutations in primary hepatocellular carcinomas of lemurs and lorises. Vet Pathol 48: 868-874, 2011.

7. Zhou XD: Recurrence and metastasis of hepatocellular carcinoma: progress and prospects. Hepatobiliary Pancreat Dis Int 1: 35-41, 2002.

8. Regina S, Rollin J, Bléchet C, Iochmann S, Reverdiau P and Gruel Y: Tissue factor expression in non-small cell lung cancer: relationship with vascular endothelial growth factor expression, microvascular density, and K-ras mutation. J Thorac Oncol 3: 689-697, 2008

9. Zuo Q, Huang H, Shi M, Zhang F, Sun J, Bin J, Liao Y and Liao W: Multivariate analysis of several molecular markers and clinicopathological features in postoperative prognosis of hepatocellular carcinoma. Anat Rec (Hoboken) 295: 423-431, 2012.

10. Hoenerhoff MJ, Chu I, Barkan D, Liu ZY, Datta S, Dimri GP and Green JE: BMI1 cooperates with H-RAS to induce an aggressive breast cancer phenotype with brain metastases. Oncogene 28: 3022-3032, 2009.

11. Lo HW: Targeting Ras-RAF-ERK and its interactive pathways as a novel therapy for malignant gliomas. Curr Cancer Drug Targets 10: 840-848, 2010.

12. Buhrman G, O'Connor C, Zerbe B, Kearney BM, Napoleon R, Kovrigina EA, Vajda S, Kozakov D, Kovrigin EL and Mattos C: Analysis of binding site hot spots on the surface of Ras GTPase. J Mol Biol 413: 773-789, 2011.

13. Nakamura M, Kitaura J,Enomoto Y, Lu Y, Nishimura K, Isobe M, Ozaki K, Komeno Y, Nakahara F, Oki T, et al: Transforming growth factor- $\beta$-stimulated clone- 22 is a negative-feedback regulator of Ras/Raf signaling: Implications for tumorigenesis. Cancer Sci 103: 26-33, 2012.

14. Lee M: Differential physiological effects of Raf-1 kinase pathways linked to protein kinase $\mathrm{C}$ activation depending on the stimulus in v-H-ras-transformed cells. Cancer Res Treat 40: 39-44, 2008

15. Yin X, Zhang BH, Qiu SJ, Ren ZG, Zhou J, Chen XH, Zhou Y and Fan J: Combined hepatocellular carcinoma and cholangiocarcinoma: clinical features, treatment modalities, and prognosis. Ann Surg Oncol: Mar 27, 2012 (Epub ahead of print). 